\title{
A Survey on Mac Protocols for Wireless Sensor Networks
}

\author{
R. Anubhama M. sc., M. phil. \\ Assistant Professor \\ Department of Computer Science \\ Bharathiar University \\ Arts and Science College \\ (Modakurichi), Sivagiri-638 109 \\ Email: anubhama.r@gmail.com
}

\begin{abstract}
Wireless Sensor Network (WSN) is an infrastructure-less wireless network of nodes that can sense the environment or physical conditions and relay the data to a sink or a gateway possibly through multiple hops. The primary task of Medium Access Control (MAC) protocols in such a network is to synchronize the task of data communication between the nodes with energy efficiency being the prime consideration due to power constraints in sensor nodes. So the most significant performance specifications for MAC protocols in WSN are throughput, efficiency, stability, fairness, low access delay, low transmission delay and low overhead. In this paper we have surveyed significant classes of MAC protocols used in WSN and reviewed the merits and demerits of those protocols based on the aforementioned specifications.
\end{abstract}

Index Terms-WSN, MAC Protocols, Throughput, Efficiency, Stability, Fairness, Low Access Delay, Low Transmission Delay, Low Overhead, Surve

\section{InTRODUCTION}

Wireless Sensor Network is a collection of sensor nodes or motes which is used to sense the data and forward it to base station(BS) [1], [2]. WSN is widely used in many applications like Military, Environmental, Forest fire detection, Flood detection, Health applications Etc. [6]. Nodes in WSN has limited resources, memory and energy. Hence, energy efficiency is an important factor in WSN sensor nodes and it must be achieved at both node level and network level [1], [4].

MAC protocols are one of the primary protocols in a network where the participating systems/nodes share a common communication medium. Conventional MAC protocols are designed to provide high throughput and QoS through better utilisation of the medium. In WSN, energy consumption of the resource constraint nodes is a key design factor but the MAC protocols designed for conventional networks are less energy efficient due to one or more of the following factors [5].

- Idle listening : A node is ready to receive but is not being sent with data

- Collisions : When two or more source nodes transfer data to the same node

\author{
Dr. T. Rajendran, M. Sc., \\ M. Phil., M. E, Ph. D, MISTE \\ Assistant Professor \\ Department of Computer Science \\ Arignar Anna Government \\ Arts College for Men, Namakkal-637002. \\ Email: rajendran_tm@yahoo.com
}

- Overhearing : Wasted effort in receiving a packet destined for another node

- Protocol Overhead : MAC related control frame structure which are non-application bytes

To counter the above factors, in WSN specific MAC protocols, researchers have proposed different variations of active/sleep mechanism combined with other techniques aimed at improving the following key attributes [7].

- Energy efficiency: Sensor nodes are mostly battery operated and are difficult to charge/change

- Latency: Time between detection of an event by sensor nodes till it reaches sink node

- Throughput: Requirement of throughput depends on specific application

Such MAC protocols can be broadly classified as follows.

\section{Contention Based}

The Contention based protocols manage the shared medium access by defining the events that must occur when two or more nodes attempt to simultaneously access the medium and by implementing rules by which a transmitting node provides scope for other nodes to transmit. They also define methods for initiating new transmissions, determining the state of the medium and managing retransmissions in the event of occupied medium. Carrier Sense Multiple Access (CSMA)/ (CSMA/CA)[3] are most common contention based MAC protocols. In CSMA/CA, the transmitter sends an RTS packet and the receiver, upon receiving it, replies with a CTS packet which refrains other nodes in the receiver's vicinity from transmitting. Although this method efficiently reduces collisions in traditional networks, in WSN, the use of RTS/CTS increases the energy consumption and supports only unicast transmissions. Hence, several variants of these contention based protocols like T-MAC[10], S-MAC[8], WiseMAC[15] were proposed for WSN. 


\section{Reservation Based}

In Reservation based protocols, each node is given a guaranteed periodic access to the shared medium by segmenting the channel into superframes and a global synchronization between nodes is assumed. A slot is reserved to each real-time node and the node uses the same slot in subsequent superframes. Time division multiple access (TDMA)[3] is a well known reservation based MAC protocol. They are more energy efficient since nodes in the network can be inactive until their allocated time slots. But the latency is directly proportional to the number of time slots and networks with large number of nodes like WSN requires a higher data rate and higher energy consumption to satisfy a deadline. Hence, several WSN specific TDMA-based MAC protocols like W-MAC[20], D-MAC[13], LL-MAC[16] were proposed.

\section{Hybrid}

The Hybrid MAC protocols combine the advantages of the TDMA and CSMA. Control packets are transmitted by random access and the data packets are transmitted in the scheduled channel. In comparison to CSMA and TDMA, the hybrid MAC protocols are energy efficient, has better scalability and improves flexibility. Some of the hybrid MAC protocols are A-MAC, IHMAC[22], IEEE 802.15.4 and Z-MAC[19].

\section{Cross Layer}

The Cross Layer MAC protocols exploits the potential synergies of the interaction among different network layers to improve the energy consumption. The B-MAC[11] and CLMAC are few examples of such protocols.

The rest of the paper presents a brief survey on few of the WSN specific MAC protocols. Section II discusses various protocols, Section III presents analysis and comparative study, Section IV discusses open issues and finally, Section $\mathrm{V}$ concludes the paper.

\section{LITERATURE REVIEW}

The Sensor-MAC (S-MAC) [8] is a CSMA based protocol in which every node follows a periodic sleep and listen time for energy efficiency. Neighboring nodes within a virtual clusters follow the same sleep/listen schedule and the neighboring nodes in two different virtual clusters follow the periods of both clusters.

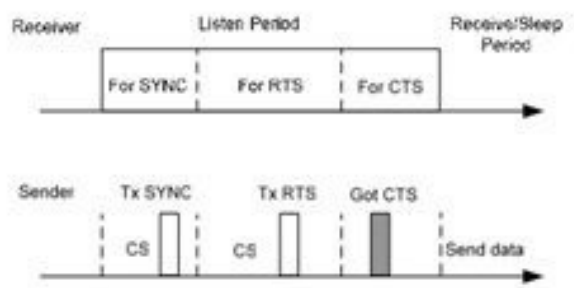

Fig 1: S-MAC with periodic sleep/listen time
Dynamic Sensor MAC (DSMAC) [9] aims to improve the latency time of S-MAC by adjusting the duty cycle of node based on the traffic and energy conditions dynamically. In DSMAC all nodes have the same duty cycle value and shared one-hop latency values in the SYNC period. When a receiver node detects the average one-hop latency value to be high, it shortens its sleep time and announces it within the SYNC period. And the sender node doubles its duty cycle after receiving this sleep period decrement signal. The latency observed with DSMAC is better than S-MAC.

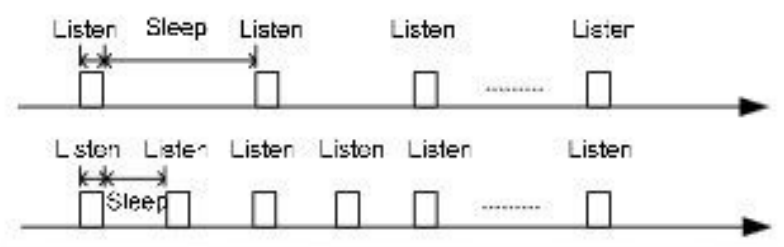

Fig 2: DSMAC with duty-cycle doubling

Timeout MAC (T-MAC) [10] improves the energy efficiency of S-MAC by reducing the listening period of sensor node during variable traffic conditions, as the nodes closer to the sink must relay more traffic. Accordingly, a node ends its listen period when no activation event has occurred for a time threshold TA.

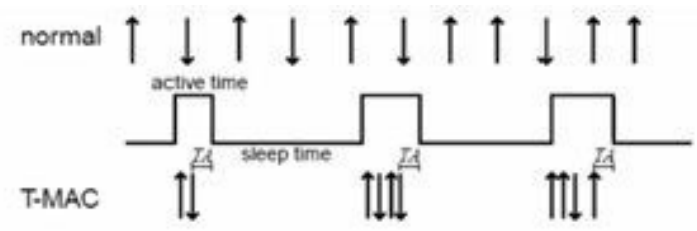

Fig 3: T-MAC with adaptive active times

In Berkeley Media Access Control (B-MAC) [11] a node self-regulates the wakeup and sleep time. The sum of awake and sleep time period is called a check interval. The sender node sends a wake-up preamble, which is not a packet but a physical layer RF pulse, greater than the check interval followed by data packet. When the receiver node wakes up, it senses the medium and if it detects the preamble, it waits for the preamble to end. If the data packet is for the node itself, it receives it otherwise goes to sleep.

Pattern MAC (PMAC) [12] is CSMA based protocol. In PMAC the wakeup and sleep time of nodes are changed dynamically based on the its own traffic pattern and that of the neighbor's.

X-MAC [14] uses the technique of strobed preamble where the sender node sends a series of short preamble packets which contain the receiver node's address. This allows the target node to interrupt the short preambles by sending acknowledgement, thus saving energy and reducing latency. 
Data gathering MAC (D-MAC)[13] is a TDMA based protocol. It is an improved Slotted Aloha protocol where slots are assigned to the nodes based on a data gathering tree. During the receive period of a node, all of its child nodes has transmit periods and subsequent slots are assigned to the nodes that are successive in the data transmission path resulting in low latency. It also uses MTS (more to send) control packets to avoid interference between different branches.

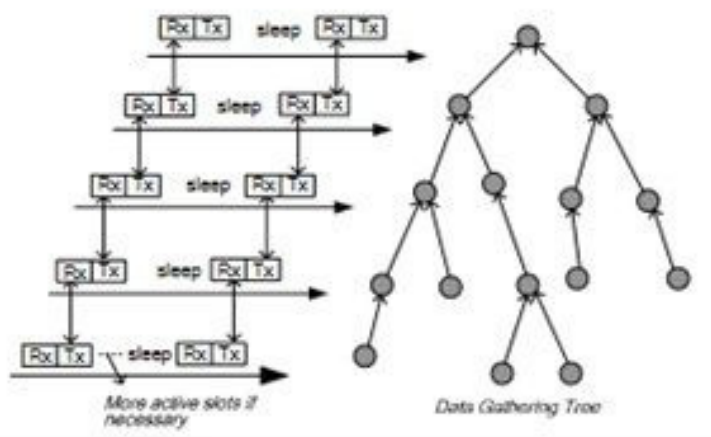

Fig 4: D-MAC and data gathering tree

Low Latency MAC (LL-MAC) [16] is TDMA based protocol designed with low latency as the primary goal. The data interval is divided into $\mathrm{X}$ divisions which in turn is divided into $\mathrm{Y}$ time slot subdivisions. Each node communicates to its parent in the time slot subdivision within the assigned division corresponding to the hop number it is in and the parent aggregates the data until its turn to communicate.

WiseMAC [15] proposes a short wakeup preamble by using the knowledge of sampling schedule of direct neighbours of the sender node.

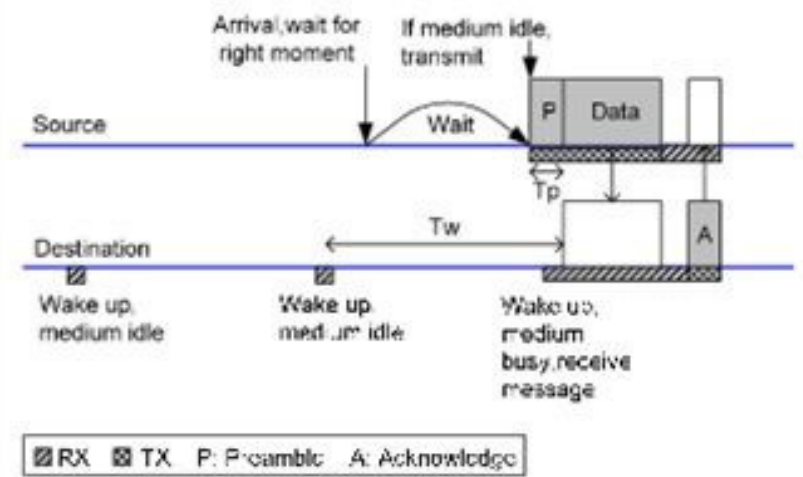

Fig 5: WiseMac

Funneling MAC [17] is a hybrid TDMA/CSMA scheme proposed to be used in the intensity region, under the control of the sink for small intensity region depths of one or two hops.

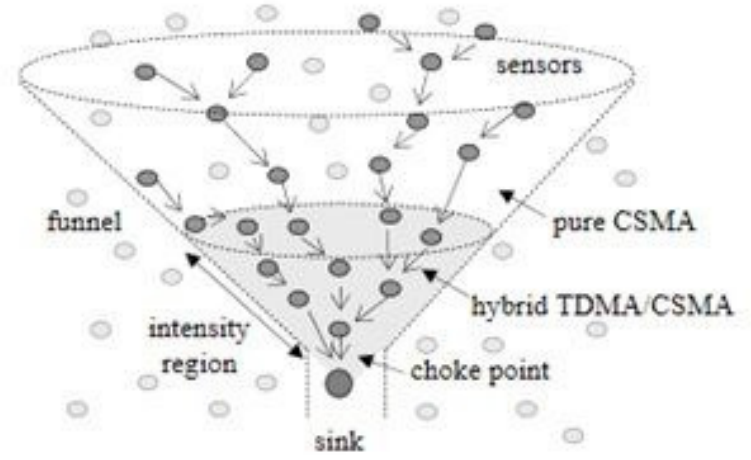

Fig 6: Concept of Funneling MAC

Traffic-adaptive MAC protocol (TRAMA) [18] is a TDMA based protocol and it uses an election algorithm to select one sender within two-hop neighborhood. In TRAMA time is divided into random-access and scheduled-access periods. The random-access period is used to establish two-hop topology information. In scheduled-access period, each node exchange its transmission schedule to its neighbours. The election algorithm is used to select the sender and receiver for the current time slot.

Zebra MAC(Z-MAC) [19] is a hybrid MAC protocol. It is a traffic adaptive protocol; in low contention it behaves like CSMA to achieve high channel utilisation and low delay, and in high contention it behaves like TDMA to achieve high channel utilisation and less collision. The efficient scheduling adjustment method is used to tolerate the network topology and data traffic variation.

Energy efficient and Quality of service aware MAC (EQ-MAC)[21] is a Hybrid MAC protocol. It differentiates the long and short messages and it uses the priority techniques for higher priority data.It uses schedule and non schedule techniques for data transmission for greater performance.

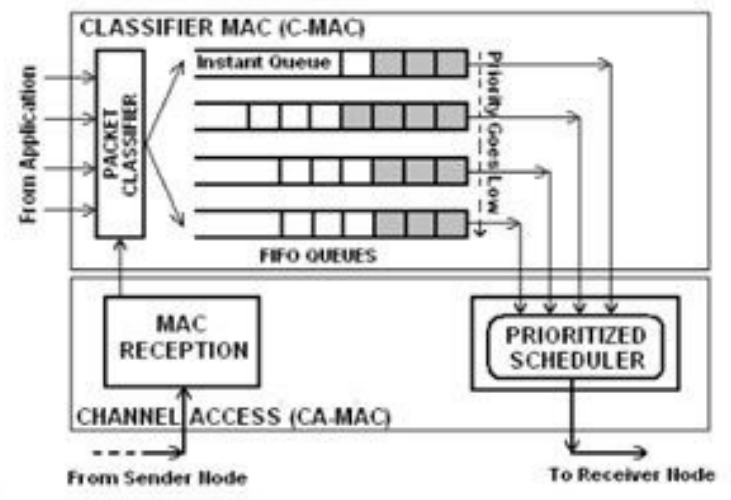

Fig 7: $E Q-M A C$ 


\section{ANALYSIS}

In this section we have analysed some of the MAC protocols discussed in the previous section for their merits and demerits.

In S-MAC[8], idle listening is reduced by periodic sleep. Bus neighboring nodes across two different clusters suffer from overhearing as they follow the sleep/wakeup cycle of both the clusters. Also the periodic sleep increases latency as most WSN routing algorithms are multi hop. Figure 8 shows the effect of number of hops on latency and throughput with and without sleep cycles.
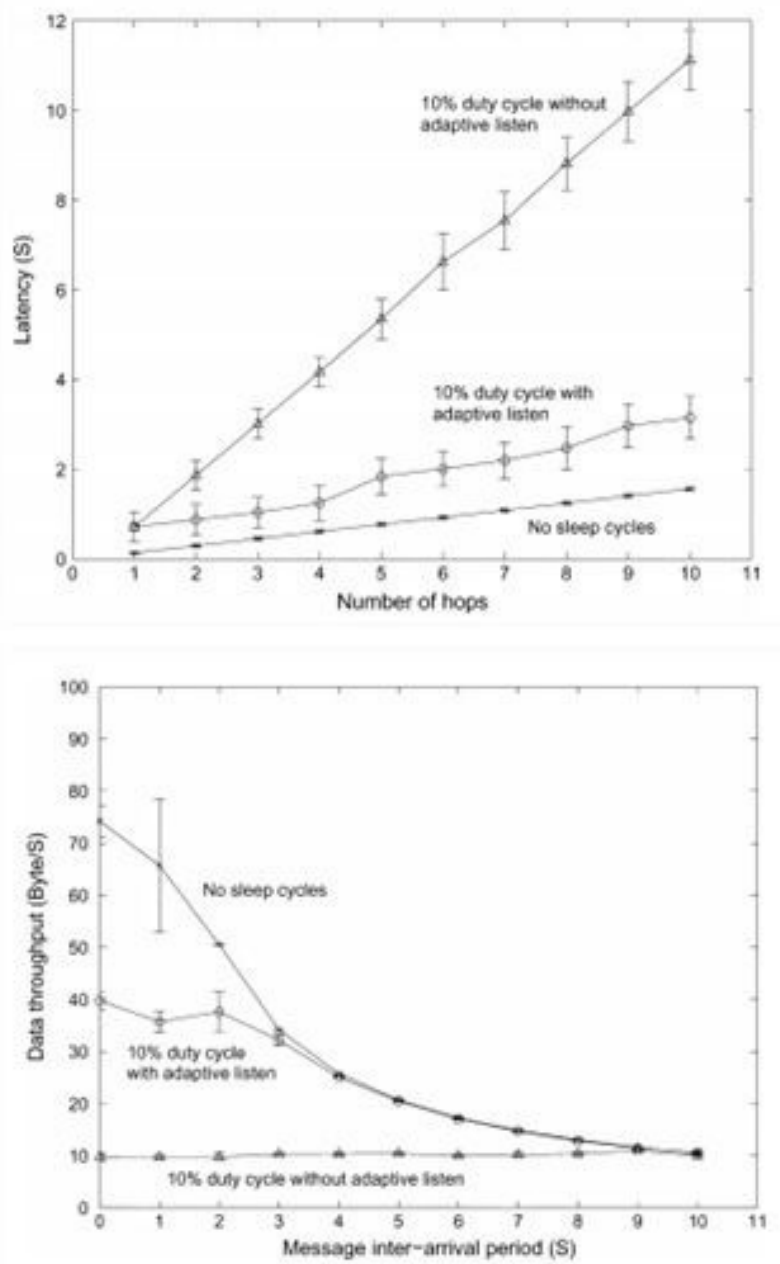

Fig 8: Latency and Throughput in S-MAC

This was based on a simple linear topology network with 11 nodes which are put in a 1-m space and are configured to send in the minimum transmission power with the source generating 20 messages, each of 100 bytes.

Although T-MAC[10] improves energy efficiency under variable traffic conditions, the synchronization of the listen periods within a virtual cluster is broken resulting in early sleeping problem. Figure 9 shows that T-MAC uses less energy than S-MAC for linear topology network

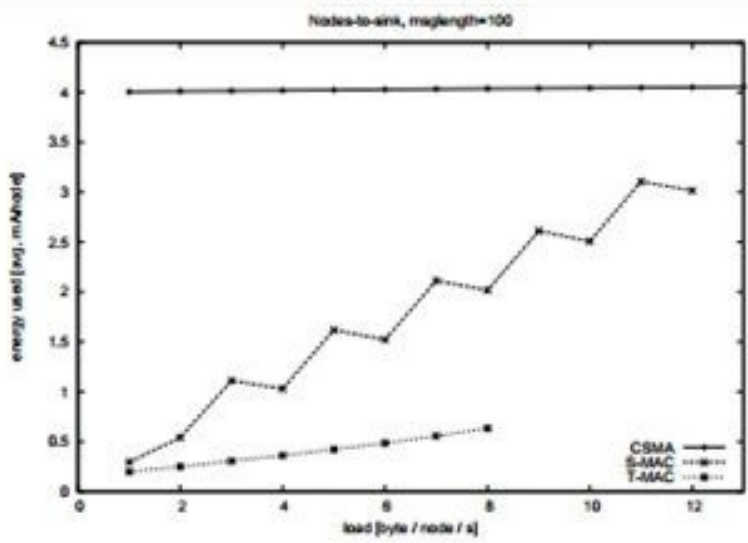

Fig 9: Energy use of T-MAC and S-MAC

D-MAC [13] fares better in terms of latency. But no collision avoidance methods were proposed for scenarios where the nodes have same schedule time.

PMAC [12] is good for relatively stable traffic conditions and performs better than S-MAC. But it is not suitable for convergecast,broadcast and point-to-point network.

B-MAC[11] is efficient at both low and high data rates and is reconfigurable by upper layers. It is also scalable to large number of nodes. But hidden terminal and multi-packet mechanisms are not provided and should be implemented by higher layers. Figure 10 shows the throughput of B-MAC against S-MAC with increased number of nodes.

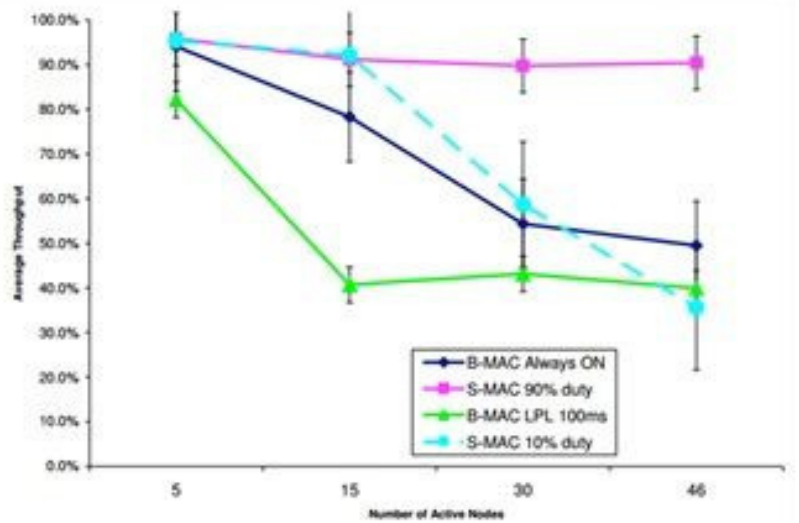

Fig 10: Throughput of B-MAC and S-MAC with different network size

For B-MAC, with a data rate of 60 seconds per packet, the average throughput was $78 \%$ for 15 nodes and $49 \%$ for 46 nodes whereas for S-MAC with $10 \%$ duty cycle and more than 15 nodes in the network, the performance degraded. 
The following table lists the comparison of various key attributes of each of the protocols discussed above along with its key merits and demerits.

\begin{tabular}{|c|c|c|c|c|c|c|}
\hline Protocol & Type & Energy & Latency & $\begin{array}{l}\text { Through } \\
\text {-put }\end{array}$ & Merits & Demerits \\
\hline S-MAC[8] & CSMA & Low & High & High & Reduced idle listening & $\begin{array}{l}\text { Predefined listen period } \\
\text { results in over-hearing during } \\
\text { variable traffic conditions }\end{array}$ \\
\hline $\begin{array}{l}\text { T-MAC } \\
{[10]}\end{array}$ & CSMA & Low & High & Low & $\begin{array}{l}\text { Handles variable traffic load } \\
\text { well }\end{array}$ & $\begin{array}{l}\text { Early sleeping affects } \\
\text { throughput }\end{array}$ \\
\hline $\begin{array}{l}\text { B-MAC } \\
{[11]}\end{array}$ & CSMA & Low & Low & High & $\begin{array}{l}\text { Good adaptability to } \\
\text { changes }\end{array}$ & Suffers from overhearing \\
\hline $\begin{array}{l}\text { WiseMAC } \\
{[15]}\end{array}$ & np-CSMA & Low & High & High & $\begin{array}{l}\text { Performs better in variable } \\
\text { traffic conditions }\end{array}$ & $\begin{array}{l}\text { Prone to hidden terminal } \\
\text { problem }\end{array}$ \\
\hline $\begin{array}{l}\text { D-MAC } \\
{[13]}\end{array}$ & TDMA & Low & Low & Low & Low latency & $\begin{array}{l}\text { Increased chances for } \\
\text { collision }\end{array}$ \\
\hline $\begin{array}{l}\text { LL-MAC } \\
{[16]}\end{array}$ & TDMA & Low & Low & Low & $\begin{array}{l}\text { Avoids hidden terminal } \\
\text { problem. }\end{array}$ & High memory usage \\
\hline $\begin{array}{l}\text { TRAMA } \\
{[18]}\end{array}$ & Hybrid & Low & Low & Low & $\begin{array}{l}\text { Performs better in } \\
\text { multicast/broadcast scenario }\end{array}$ & High duty cycle value \\
\hline $\begin{array}{l}\text { W-MAC } \\
{[20]}\end{array}$ & TDMA & Low & Low & Low & Tolerates traffic variation & $\begin{array}{l}\text { Does not support concurrent } \\
\text { transmission }\end{array}$ \\
\hline
\end{tabular}

\section{OPEN ISSUES}

While TDMA[3] based protocols has the main advantage of collision-free medium access, clock drift problem and decreased throughput at low traffic loads are open issues which are being addressed by researchers. In WSN, these TDMA protocols have the additional challenge of adaptation to topology changes caused by broken links due to battery exhaustion, insertion of new nodes, sleep/wakeup schedules of relay nodes and clustering algorithms. While CSMA[3] methods offer lower delay and good throughput at lower traffic loads, additional collision avoidance or collision detection are required to handle the collision possibilities. CDMA based protocols offers collision-free medium, but they require high computational power which virtually rules them out of consideration for energy sensitive systems like WSN. There are open issues to prove that the collision-free medium offered by these protocols can be a tradeoff for energy consumption caused by high computational power. And comparison of CSMA, TDMA and other MAC protocols under a common framework is still an open research area. Also, rating these protocols based on not just the layer 2 performance but the overall system performance is still lacking or insufficient, which can provide a greater push for multi-layer protocols.

With respect to specific protocols discussed in this paper, in S-MAC [8], adaptability to the changes in network topology requires more work. TMAC discusses virtual clustering but it is not clearly described. PMAC[12] is not suitable for point to point converge gate and broadcast based network. In D-MAC[13] the sensor nodes are fixed based on assumption and strength of sensor nodes are not considered. In X-MAC[14] only few number of nodes can be used but latency can be measured with more data points, so the research can be carried out with more number of nodes. In W-MAC[20] transmissions are not carried-out simultaneously, but if we reuse the same time slot again we can support concurrent transmissions.

\section{CONCLUSION}

This paper presents the study of various WSN specific MAC protocols based on various design factors. It must be highlighted that there is no one protocol accepted as a universal standard. The prime reason is that the choice of the MAC protocol in WSN will be application specific based on the requirement of the key attributes specific to that application. Another reason is that the lower layers lack standardisation and similar conclusion can be drawn for upper layers as well. Hence, a cross-layer design approach is still feasible as attempted in few of the protocols discussed in this paper and it seems to be a promising research area which has to be studied more extensively. 


\section{REFERENCES}

[1] Praveen Lalwani, Haider Banka, Chiranjeev kumar, "BERA A Biogeography based Energy Saving Routing Architecture for Wireless Sensor Networks", Soft computing, Springer, November 2016.

[2] Praveen Lalwani, Haider Banka, Chiranjeev kumar,"CRWO:Cluste Head Selection and Routing in Wireless Sensor Networks using optics inspired optimization",Peer-to-Peer Networking and Applications, Springer October 2016

[3] Pallavi Meshram, and M. V. Nimbalkar, "A Survey on Energy Efficient MAC Protocols with Differentiation in Traffic for Wireless Sensor Networks", International Journal of Science and Research (IJSR), ISSN (Online): 2319-7064 Index Copernicus Value (2013) 6.14 | Impact Factor (2015): 6.391.

[4] Ravi T. Matani and Tejas M. Vasavada, "A Survey on MAC Protocols for Data Collection in Wireless Sensor Networks",International Journal of Computer Applications (0975 - 8887) Volume 114 - No. 6 , March 2015

[5] Rohan Parmar and Dr. R. C. Poonia, "A Literature Survey on Energy Efficient MAC Protocols for WSN", International Journal of Computer Science and Information Technologies, Vol. 5 (3), 2014, 3421-3424,2016.

[6] Sandeep Verma,Richa Mehta,Divya Sharma and Kanika Sharma, "Wireless sensor network and hierarchical routing protocols: A review", International Journal of Computer Trends and Technology (IJCTT), volume 4 Issue 8, August 2013.

[7] Kshama D. Pandya, Tejas Vasavada, "Data collection in tree-based wireless sensor network using TDMA scheduling", International Journal of Advance Research in Computer Science and Software Engineering, volume 4, Issue 3, March 2014.

[8] Wei YE, John HEIDEMANN and Deborah ESTRIN, "An EnergyEfficient MAC Protocol for Wireless Sensor Networks", In Proceedings of the 21st International Annual Joint Conference of the IEEE Computer and Communications Societies, INFOCOM 02, New York, USA, pp. 1567-1576,June 2002.

[9] P. Lin, C. Qiao and X. Wang, "Medium access control with a dynamic duty cycle for sensor networks", IEEE Wireless Communications and Networking Conference, Volume: 3, Pages: 1534 - 1539, March 2004.

[10] Dam T. and Langendoen K, "An adaptive energy-efficient MAC protocol for wireless sensor networks", The 1st ACM Conference on Embedded Networked Sensor Systems (Sensys,,03), Los Angeles, CA, USA, November, 2003

[11] Joseph Polastre, Jason Hill and David Culler,"Versatile low power media access for wireless sensor networks", 2nd ACM Conference on Embedded Networked Sensor Systems (SenSys). Baltimore, MD, USA, ACM Press vol. 2nd, pp. 95-107, November 2004.
[12] T. Zheng, S.Radhakrishnan and V.Sarangan, "PMAC: An adaptive energy-efficient MAC protocol for Wireless Sensor Networks", Parallel and Distributed Processing Symposium, 2005. Proceedings. 19th IEEE International, pp4-8, April 2005.

[13] G. Lu, B. Krishnamachari and C. S. Raghavendra,"An adaptive Energy-Efficient and Low-Latency MAC for Data Gathering in wireless Sensor Networks" ,In Proceedings of the 18th International Parallel and Distributed Processing Symposium, IPDPS 04, Santa Fe, NM, USA, pp. 224-231, April 2004

[14] Michael Buettner, Gary V. Yee, Eric Anderson, and Richard Han, "Xmac: A short preamble MAC protocol for duty-cycled wireless sensor networks" ,International conference on Embedded networked sensor systems (SenSys) vol. 4th, 2006.

[15] Amre El-Hoiydi and Jean-Dominique Decotignie, "Wisemac: An ultra-low power mac protocol for the multihop wireless sensor networks", Lecture Notes in Computer Science(LNCS) Vol.3121, pp. $18-31,2004$

[16] I. Marin, J. Arias, E. Arceredillo, A. Zuloaga, I. Losada and J. Mabe, "LL-MAC: A low latency MAC protocol for wireless self-organised networks", Microprocessors Microsystems, volume 32, pp 197-209, 2008.

[17] Gahng-Seop Ahn,Emiliano Miluzzo, Andrew T. Campbell, Se Gi Hong and Francesca CuomoAhn, "Funneling-MAC: A localized, sinkoriented MAC for boosting fidelity in sensor networks", In Proceedings the 4th ACM Conference on Embedded Networked Sensor Systems, SENSYS 06, Boulder, USA, pp. 293-306, October November 2006.

[18] Venkatesh Rajendran, Katia obraczka and J. J. Garcia luna aceves, "Energy-efficient, collision-free medium access control for wireless sensor networks", In Proceedings of the 1st ACM Conference on Embedded Networked Sensor Systems, SENSYS" 03, Los Angeles, CA, USA, pp. 181-192,November 2003

[19] Injong Rhee, Ajit warrier, mahesh aia,jeongki min, Mihail L.sichitiu, "Z-MAC: a Hybrid MAC for wireless sensor networks", SenSys 05, pp 2-4, November 2005 .

[20] M. Xia, Dong.Y and Lu D, "W-MAC: A Workload-Aware MAC Protocol for Heterogeneous Convergecast in Wireless Sensor Networks", in Sensors 2011, pp. 2505-2524; doi: 10.3390/ s110302505,2011.

[21] Ilker Demirkol, Cem Ersoy and Fatih Alagöz, "Mac protocols for wireless sensor networks: A survey",IEEE Commun. Mag. Vol. 06, pp. 115-121, April 2006.

[22] M. Arifuzzaman, M. Matsumoto, and T. Sato, "An Intelligent Hybrid MAC with Traffic- Differentiation Based QoS for wireless Sensor Networks," Sensors Journal, IEEE, vol. 13, no. 6, pp. 2391-2399, 2013 